\title{
SYM 2206 (a potent non-competitive AMPA receptor antagonist) elevates the threshold for maximal electroshock-induced seizures in mice
}

\author{
JAROgNiEW J. LUSZCZKI ${ }^{1,2^{*}}$, MAgDALENA LeSZKOWICZ ${ }^{1}$, \\ Maria W. Kondrat-WrobeL ${ }^{1}$, Magdalena FloreK-LuszczKi ${ }^{3}$
}

\begin{abstract}
${ }^{1}$ Department of Pathophysiology, Medical University of Lublin, Ceramiczna 1, 20-150 Lublin, Poland
${ }^{2}$ Isobolographic Analysis Laboratory, Institute of Rural Health, Jaczewskiego 2, 20-950 Lublin, Poland

${ }^{3}$ Department of Public Health, Institute of Rural Health, Jaczewskiego 2, 20-950 Lublin, Poland
\end{abstract}

\begin{tabular}{l}
\hline ARTICLE INFO \\
\hline Received 26 March 2014 \\
Accepted 27 May 2014 \\
\hline
\end{tabular}

Keywords:

SYM 2206,

threshold for maximal

electroshock-induced seizures,

$\mathrm{TID}_{20}$,

$\mathrm{TID}_{50}$,

mice.

\begin{abstract}
The aim of this study was to determine the effect of SYM 2206 (a potent non-competitive AMPA receptor antagonist) on the threshold for maximal electroshock (MEST)-induced seizures in mice. Electroconvulsions were produced in mice by means of a current (sinewave, $50 \mathrm{~Hz}$, maximum $500 \mathrm{~V}$, strength from 4 to $14 \mathrm{~mA}, 0.2$-s stimulus duration, tonic hind limb extension taken as the endpoint) delivered via ear-clip electrodes. SYM 2206 administered systemically (i.p.), $30 \mathrm{~min}$ before the MEST test, at doses of $2.5 \mathrm{and} 5 \mathrm{mg} / \mathrm{kg}$, did not alter the threshold for maximal electroconvulsions in mice. In contrast, SYM 2206 at doses of 10 and $20 \mathrm{mg} / \mathrm{kg}$ significantly elevated the threshold for maximal electroconvulsions in mice $(\mathrm{P}<0.01$ and $\mathrm{P}<0.001)$. Linear regression analysis of SYM 2206 doses and their corresponding threshold increases allowed for the determination of threshold increasing doses by $20 \%$ and $50 \%$ (TID $_{20}$ and $\mathrm{TID}_{50}$ values) that elevate the threshold in drug-treated animals over the threshold in control animals. The experimentally derived TID $_{20}$ and TID $_{50}$ values for SYM 2206 were 4.25 and $10.56 \mathrm{mg} / \mathrm{kg}$, respectively. SYM 2206 dose-dependently increased the threshold for MEST-induced seizures, suggesting the anticonvulsant action of the compound in this seizure model in mice.
\end{abstract}

\section{INTRODUCTION}

It is widely accepted that alpha-amino-3-hydroxy5-methyl-4-isoxazolepropionic acid (AMPA) receptors are key mediators of seizure spread in the central nervous system and play an important role in epileptogenesis and in seizure-induced brain damage $[4,15,16]$. There is emerging evidence suggesting that AMPA receptor antagonists could have broad utility in epilepsy therapy and represent promising targets for antiepileptic drugs (AEDs) $[4,15]$. Experimental studies have revealed that both, competitive and noncompetitive AMPA receptor antagonists are broad-spectrum anticonvulsants in animal seizure models and these antagonists can potentiate the antiseizure activity of conventional AEDs [15].

\footnotetext{
Corresponding author

e-mail: jarogniew.luszczki@gmail.com, jluszczki@yahoo.com tel: +4881 718-73-65,

fax: $+4881718-73-64$
}

The anticonvulsant potential of agents or drugs in the maximal electroshock-induced seizure threshold (MEST) test can be readily determined by denoting doses increasing the threshold by $20 \%$ and $50 \%$ (TID $_{20}$ and $\mathrm{TID}_{50}$ values) $[6,7,17]$. These doses elevate the threshold for electroconvulsions in drug-treated animals by $20 \%$ and $50 \%$ over the threshold in control animals $[6,7,17]$. To determine the $\operatorname{TID}_{20}$ and $\mathrm{TID}_{50}$ values in preclinical studies, linear regression analysis of drug doses and their corresponding threshold values is used $[6,7,17]$. Recently, we have reported that some second-generation AEDs (i.e., gabapentin, levetiracetam, stiripentol, tiagabine and vigabatrin) increased the threshold for electroconvulsions in mice that allowed the calculation of $\operatorname{TID}_{20}$ and $\operatorname{TID}_{50}$ values $[9,10,12,13]$.

SYM $2206[( \pm)-4-(4-a m i n o p h e n y l)-1,2$-dihydro1-methyl-2-propylcarbamoyl-6,7-methylenedioxy-phthalazine] is a potent non-competitive AMPA receptor antagonist that exerted anticonvulsant effects in various in vitro and in vivo studies $[1,2,14,18]$. Therefore, the aim this study was to determine the TID $_{20}$ and TID $_{50}$ values for SYM 2206 in the MEST test. 


\section{MATERIALS AND METHODS}

Animals and experimental conditions. All experiments were performed on adult male Swiss mice weighing 22-26 g. The animals were purchased from a licensed breeder (Dr. J. Kolacz, Warszawa, Poland). The mice were kept in colony cages with free access to food and tap water, under standardized housing conditions (natural light-dark cycle, temperature was $21 \pm 1^{\circ} \mathrm{C}$ ). After 7 days of adaptation to laboratory conditions, the animals were randomly assigned to experimental groups consisting of 8 mice. Each mouse was used only once. All tests were performed between 9.00 a.m. and 2.00 p.m. Procedures involving animals and their care were conducted in conformity with current European Community and Polish legislation on animal experimentation. Additionally, all efforts were made to minimize animal suffering and to use only the number of animals necessary to produce reliable scientific data. The experimental protocols and procedures listed below were conformed to the Guide for the Care and Use of Laboratory Animals and approved by the Second Local Ethics Committee at the University of Life Sciences in Lublin (license no.: 12/2012). The total number of animals used in this study was 160 .

Drug. SYM 2206 [( \pm )-4-(4-Aminophenyl)-1,2-dihydro1-methyl-2-propylcarbamoyl-6,7-methylenedioxyphthalazine] (Tocris Bioscience, Bristol, United Kingdom) was suspended in a $1 \%$ solution of Tween 80 (Sigma, St. Louis, MO, USA) in distilled water and administered intraperitoneally (i.p.) in a volume of $0.005 \mathrm{ml} / \mathrm{g}$ body weight at $30 \mathrm{~min}$ before MEST-induced seizures.

Maximal electroshock seizure threshold (MEST) test. Electroconvulsions were produced by means of an alternating current (sine-wave, $0.2 \mathrm{~s}$ stimulus duration, $50 \mathrm{~Hz}$, maximum stimulation voltage of $500 \mathrm{~V}$, current strength ranging from 4 to $14 \mathrm{~mA}$ ) delivered via ear-clip electrodes by a Rodent Shocker generator (Type 221, Hugo Sachs Elektronik, Freiburg, Germany). The electrical system of the stimulator was self-adjustable so that changes in impedance did not result in alterations of current intensity (i.e., the system provides constant current stimulation). The criterion for the occurrence of seizure activity was the tonic hind limb extension (i.e., the hind limbs of animals outstretched $180^{\circ}$ to the plane of the body axis). To evaluate the threshold for maximal electroconvulsions, at least 4 groups of mice, consisting of 8 animals per group, were challenged with electroshocks of various intensities to yield 10-30\%, $30-50 \%, 50-70 \%$, and $70-90 \%$ of animals with seizures. Then, a current intensity-response curve was constructed, according to a log-probit method by Litchfield and Wilcoxon [5], from which a median current strength $\left(\mathrm{CS}_{50}\right.$ in $\left.\mathrm{mA}\right)$ was calculated. Each $\mathrm{CS}_{50}$ value represents the current intensity required to induce tonic hind limb extension in 50\% of the mice challenged. The $\mathrm{CS}_{50}$ values with their $95 \%$ confidence limits were calculated by computer log-probit analysis according to Litchfield and Wilcoxon [5]. Subsequently, the $95 \%$ confidence limits were transformed to their S.E.M. according to the procedure described in our earlier study $[8,11]$. Again, after administration of a single dose of SYM 2206 to 4 groups of animals, the mice were subjected to electroconvulsions (each group with a constant current intensity). The threshold for maximal electroconvulsions was recorded for 4 different doses of SYM 2206 i.e., 2.5, 5, 10 and $20 \mathrm{mg} / \mathrm{kg}$, which were empirically selected from a pilot study. Statistical analysis of $\mathrm{CS}_{50}$ values was performed with one-way ANOVA followed by the post-hoc TukeyKramer test for multiple comparisons. Subsequently, the percentage of increase in $\mathrm{CS}_{50}$ values for animals injected with increasing doses of SYM 2206 over the control (vehicle-treated animals) was calculated. The doses of SYM 2206 and their resultant percentage of threshold increase over the control (vehicle-treated animals) were graphically plotted in rectangular coordinates of the Cartesian plot system and examined with least-squares linear regression analysis [3]. From linear regression equation, the $\mathrm{TID}_{20}$ and $\mathrm{TID}_{50}$ values were determined as recommended by Löscher et al. [6] and Swinyard et al. [17]. The experimental procedure has been described in more detail in our earlier studies $[9,10,12,13]$. Statistical evaluation of data was performed using commercially available GraphPad Prism 5 (GraphPad Software Inc., San Diego, CA, USA). Differences between the respective values were statistically significant at $\mathrm{P}<0.05$.

\section{RESULTS}

Effect of SYM 2206 on the threshold for maximal electroshock-induced seizures.

SYM 2206 administered systemically (i.p., 30 min prior to the test), at doses of 2.5 and $5 \mathrm{mg} / \mathrm{kg}$ did not affect the threshold for maximal electroconvulsions in mice (Tab.1).

Table 1. Effect of SYM 2206 on the threshold for electroconvulsions in mice

\begin{tabular}{|c|c|c|c|}
\hline Treatment $(\mathrm{mg} / \mathrm{kg})$ & $\mathrm{CS}_{50}(\mathrm{~mA})$ & $\mathrm{n}$ & TI (\%) \\
\hline Vehicle & $5.58 \pm 0.41$ & 24 & - \\
\hline SYM 2206 (2.5) & $6.01 \pm 0.53$ & 16 & 7.7 \\
\hline SYM 2206 (5) & $7.12 \pm 0.56$ & 24 & 27.6 \\
\hline SYM 2206 (10) & $8.27 \pm 0.43 * *$ & 16 & 48.2 \\
\hline SYM 2206 (20) & $10.82 \pm 0.47^{* * *}$ & 24 & 93.9 \\
\hline \multicolumn{4}{|c|}{$F(4 ; 99)=19.81 ; P<0.0001$} \\
\hline
\end{tabular}

Data are presented as median current strengths $\left(\mathrm{CS}_{50}\right.$ values in $\mathrm{mA} \pm$ S.E.M.) required to evoke seizure activity (tonic hindlimb extension) in $50 \%$ of animals tested in the maximal electroshock seizure threshold (MEST) test. The $\mathrm{CS}_{50}$ values were calculated according to the log-probit method by Litchfield and Wilcoxon [5]. SYM 2206 was administered systemically (i.p.) at $30 \mathrm{~min}$ before the initiation of electroconvulsions in mice. The threshold for control (vehicle-treated) animals was considered as a baseline (reference) value for calculations of percentage in the threshold increase (TI) following SYM 2206 administration. Statistical analysis of data was performed with one-way ANOVA followed by the post-hoc Tukey-Kramer test for multiple comparisons. TI - threshold increase in $\%$ (the control value of $5.58 \mathrm{~mA}$ was considered as $100 \%$ ); $\mathrm{n}$ - number of animals tested at those current strength intensities, whose seizure effects ranged between $16 \%$ and $84 \%$; F - F-statistics from one-way ANOVA; $\mathrm{P}-$ probability value from one-way ANOVA.

In this case, the experimentally derived $\mathrm{CS}_{50}$ values for animals receiving SYM 2206 (2.5 and $5 \mathrm{mg} / \mathrm{kg}$ ) did not differ significantly from the $\mathrm{CS}_{50}$ value as determined for control animals in the MEST test in mice (Tab.1). In contrast, SYM 2206 administered at doses of 10 and $20 \mathrm{mg} / \mathrm{kg}$ significantly elevated the threshold for maximal electroconvulsions in mice $(\mathrm{P}<0.01$ and $\mathrm{P}<0.001$, respectively; 
one-way ANOVA: $F(4 ; 99)=19.81 ; P<0.0001$; Tab. 1$)$. It was found that SYM 2206 at doses of 2.5, 5, 10 and $20 \mathrm{mg} / \mathrm{kg}$ elevated the threshold for MEST-induced clonic seizures by $7.7 \%, 27.6 \%, 48.2 \%$ and $93.9 \%$, respectively (Tab.1, Fig.1). The experimentally derived $\mathrm{TID}_{20}$ and $\mathrm{TID}_{50}$ values for SYM 2206 were 4.25 and $10.56 \mathrm{mg} / \mathrm{kg}$, respectively, in the MEST test in mice (Fig. 1).

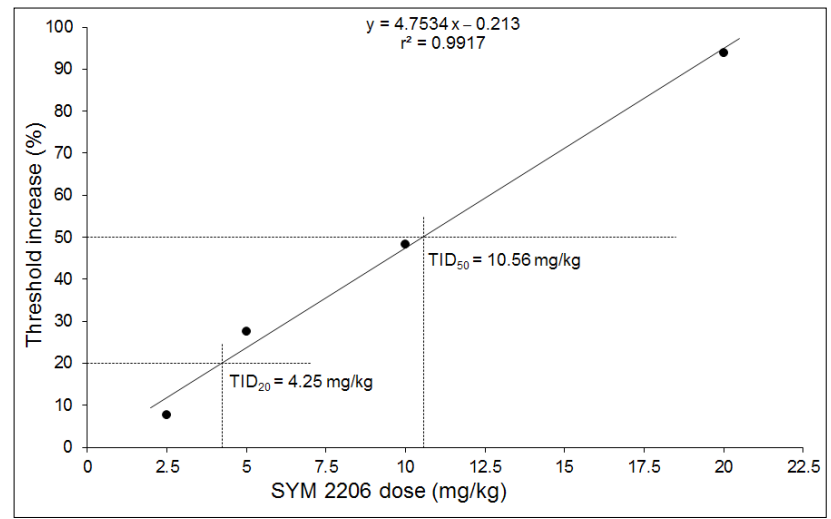

Points placed on the graph represent threshold increasing doses of SYM 2206, which were experimentally denoted in the MEST test in mice. Linear regression analysis allowed for the determination of equation for dosethreshold increase relation for SYM 2206, as follows: $y=4.7534 \mathrm{x}-0.213$ $\left(\mathrm{r}^{2}=0.9917\right)$; where $\mathrm{y}$ - threshold increase in $\%, \mathrm{x}$ - the dose of SYM 2206, and $\mathrm{r}^{2}-$ coefficient of determination [3]. From this equation one denotes the $\mathrm{TID}_{20}$ and $\mathrm{TID}_{50}$ (threshold increasing doses by $20 \%$ and $50 \%$ ) for the MEST test. In this study, these values were 4.25 and $10.56 \mathrm{mg} / \mathrm{kg}$, respectively. SYM 2206 was administered i.p., 30 min before the threshold evaluation

Figure 1. Dose-threshold increase relation for SYM 2206 in the maximal electroshock seizure threshold (MEST) test in mice

\section{DISCUSSION}

Results presented herein clearly indicate that SYM 2206 (at doses of 10 and $20 \mathrm{mg} / \mathrm{kg}$ ) significantly elevated the threshold for maximal electroconvulsions in mice. Linear regression analysis of SYM 2206 doses and their corresponding threshold increasing values over the threshold in control animals in the MEST test revealed that there was a close relation between the doses of SYM 2206 and their biological effects in terms of seizure suppression in mice (Tab. 1, Fig. 1). In the MEST test in mice, the experimentally-derived TID $_{20}$ and TID $_{50}$ values for SYM 2206 were 4.25 and $10.56 \mathrm{mg} / \mathrm{kg}$, respectively.

Results obtained for SYM 2206 in the MEST test can be readily compared to those reported earlier for some second-generation AEDs (Tab.2). It has been documented that gabapentin, levetiracetam, stiripentol, tiagabine and vigabatrin increased the threshold for electroconvulsions in mice $[9,10,12,13]$. The comparison of TID $_{50}$ values of the second-generation AEDs with those calculated for SYM 2206 revealed that only tiagabine had the $\mathrm{TID}_{50}$ value lower than that of SYM 2206 (Tab.2). In contrast, the TID values denoted for gabapentin, levetiracetam, stiripentol and vigabatrin were considerably higher than those denoted for SYM 2206 in this study (Tab.2). Thus, it can be ascertained that SYM 2206 possesses a strong anticonvulsant action in the MEST model in mice.

Table 2. Characteristics of various second-generation antiepileptic drugs in the MEST test in mice

\begin{tabular}{|l|c|c|c|}
\hline \multicolumn{1}{|c|}{ Drug } & $\mathrm{TID}_{20}$ & $\mathrm{TID}_{50}$ & References \\
\hline Gabapentin & 70.0 & 112.7 & {$[12]$} \\
\hline Levetiracetam & 44.0 & 150.0 & {$[9]$} \\
\hline Stiripentol & 103.2 & 195.8 & {$[13]$} \\
\hline SYM 2206 & 4.25 & 10.56 & [present study] \\
\hline Tiagabine & 4.4 & 8.0 & {$[10]$} \\
\hline Vigabatrin & 226.2 & N.D. & {$[10,12]$} \\
\hline
\end{tabular}

Results are presented as doses of the drugs increasing the threshold for electroconvulsions by $20 \%$ and $50 \%\left(\mathrm{TID}_{20}\right.$ and $\mathrm{TID}_{50}$ in $\mathrm{mg} / \mathrm{kg}$ ), which were experimentally denoted in the MEST test in mice. N.D. - not determined.

\section{CONCLUSION}

Based on this preclinical study, it can be ascertained that SYM 2206, in a dose-dependent manner, suppresses MESTinduced tonic seizures in mice. If the results from this study could be extrapolated to clinical settings, SYM 2206 would become a candidate as a potential new drug treatment in epilepsy.

\section{ACKNOWLEDGEMENTS}

This study was supported by grants from Medical University of Lublin and Institute of Rural Health (Lublin, Poland). Professor J.J. Luszczki is a Member of the Academy of Young Scientists (Polish Academy of Sciences, Warszawa, Poland).

\section{DISCLOSURE OF CONFLICTS OF INTEREST}

The authors have no disclosures to declare.

\section{REFERENCES}

1. Behr J. et al.: Kindling enhances kainite receptor-mediated depression of GABAergic inhibition in rat granule cells. Eur. J. Neurosci., 16, 861, 2002.

2. Ghersi C. et al.: Pharmacological heterogeneity of release-regulating presynaptic AMPA/kainate receptors in the rat brain: study with receptor antagonists. Neurochem. Int., 42, 283, 2003.

3. Glantz S.A., Slinker B.K.: editors (2001). Primer of applied regression and analysis of variance, second edition. McGraw-Hill Inc.: New York, NY.

4. Krauss G.L.: Perampanel: a selective AMPA antagonist for treating seizures. Epilepsy Curr., 13, 269, 2013.

5. Litchfield J.T., Wilcoxon F.: A simplified method of evaluating doseeffect experiments. J. Pharmacol. Exp. Ther., 96, 99, 1949.

6. Löscher W., Fassbender C.P., Nolting B.: The role of technical, biological and pharmacological factors in the laboratory evaluation of anticonvulsant drugs. II. Maximal electroshock seizure models. Epilepsy Res., 8, 79, 1991.

7. Löscher W., Wauquier A.: Use of animal models in developing guiding principles for polypharmacy in epilepsy. Epilepsy Res., Suppl. 11, 61, 1996.

8. Łuszczki J.J., Antkiewicz-Michaluk L., Czuczwar S.J.: Isobolographic analysis of interactions between 1-methyl-1,2,3,4-tetrahydroisoquinoline and four conventional antiepileptic drugs in the mouse maximal electroshock-induced seizure model. Eur. J. Pharmacol., $602,298,2009$.

9. Łuszczki J.J., Czuczwar S.J.: How significant is the difference between drug doses influencing the threshold for electroconvulsions? Pharmacol. Rep., 57, 782, 2005. 
10. Łuszczki J.J., Czuczwar S.J.: Isobolographic characterization of interactions between vigabatrin and tiagabine in two experimental models of epilepsy. Prog. Neuropsychopharmacol. Biol. Psychiatry, 31, 529, 2007.

11. Łuszczki J.J. et al.: Isobolographic analysis of interactions between loreclezole and conventional antiepileptic drugs in the mouse maximal electroshock-induced seizure model. NaunynSchmiedebergs Arch. Pharmacol., 373, 169, 2006.

12. Łuszczki J.J. et al.: Isobolographic and behavioral characterizations of interactions between vigabatrin and gabapentin in two experimental models of epilepsy. Eur. J. Pharmacol., 595, 13, 2008.

13. Łuszczki J.J. et al.: Stiripentol in a dose-dependent manner elevates the threshold for maximal electroshock-induced seizures in mice. J. Pre-Clin. Clin. Res., 1, 155, 2007.
14. Pelletier J.C. et al.: Substituted 1,2-dihydrophthalazines: potent, selective, and noncompetitive inhibitors of the AMPA receptor. J. Med. Chem., 39, 343, 1996

15. Rogawski M.A.: AMPA receptors as a molecular target in epilepsy therapy. Acta Neurol. Scand. Suppl., 197, 9, 2013.

16. Rogawski M.A., Donevan S.D.: AMPA receptors in epilepsy and as targets for antiepileptic drugs. Adv. Neurol., 79, 947, 1999.

17. Swinyard E.A., Brown W.C., Goodman L.S.: Comparative assays of antiepileptic drugs in mice and rats. J. Pharmacol. Exp. Ther., 106, 319, 1952

18. Welch N.C. et al.: Traditional AMPA receptor antagonists partially block Na v1.6-mediated persistent current. Neuropharmacology, 55, 1165,2008 